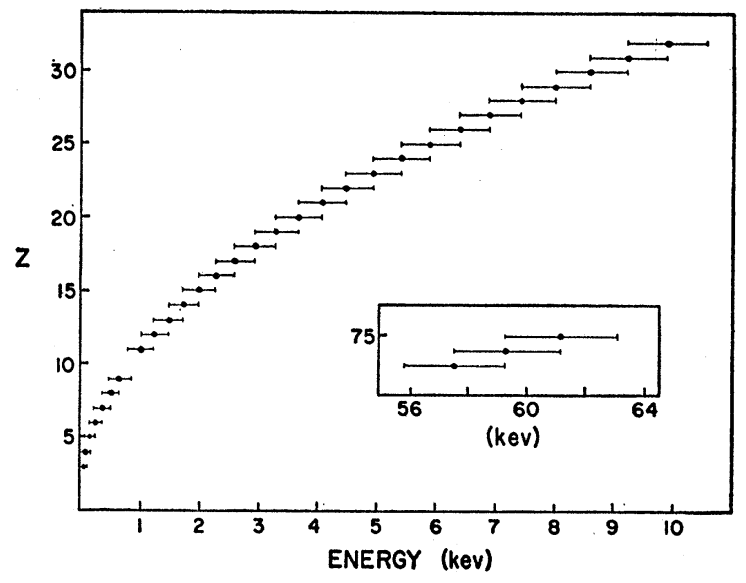

FIG. 1. $K \alpha$ energies and calculated PHD half-widths as a function of atomic number, based on 15 percent half-width for ${ }_{29} \mathrm{Cu}$. Insert shows the half-widths for the $K \alpha$ of ${ }_{73} \mathrm{Ta},{ }_{74} \mathrm{~W}$, and ${ }_{75} \mathrm{Re}$.

A more generally used quantity is the "percent halfwidth" which is the ratio of the half-width to the pulse amplitude at maximum rate $(\times 100)$.

From an extension of some work by Frisch $^{2}$ and Fano $^{3}$ on the statistical fluctuations in multiplicative processes, it was found for the peaks in the PHD of a proportional counter, that

$$
\text { (half-width) } \propto \text { (energy })^{\frac{1}{2}} .
$$

This has been experimentally verified ${ }^{4}$ in this Laboratory using rare-gas filled proportional counters to detect $\mathrm{x}$-radiations ranging in energy from 5 to $50 \mathrm{kev}$. Although radiations below $5 \mathrm{kev}$ have not been directly checked, the escape pulses from the counter offered a means of extending this range into the soft $\mathrm{x}$-ray region to $1 \mathrm{kev}$.

It is interesting to note that Moseley's Law, giving the relation between energy of characteristic lines and atomic number,

$$
(Z-\sigma) \propto(\text { energy })^{\frac{1}{2}}
$$

has a similar form. Two consequences follow: (a) The half-width of the PHD produced by the characteristic radiation of an element is approximately proportional to the atomic number of the element. The percent halfwidth, therefore, is a means of identifying elements, provided the half-width for a known energy has been determined. (b) The ability of a proportional counter to resolve characteristic $x$-radiations of adjacent elements is approximately constant over all the elements. That is,

$$
B(Z) /[E(Z+1)-E(Z)] \cong \text { const, }
$$

where $B(Z)$ is the half-width of the PHD given by x-rays from an emitter of atomic number $Z$, and $E(Z)$ is the energy of the $x$-rays.

In order to determine the degree of approximation and the validity of (b), Eqs. (1) and (2) are combined:

$$
B(Z) \propto[E(Z)]^{\frac{1}{2}} \propto(Z-\sigma) .
$$

Then,

$$
\begin{aligned}
& \frac{B(Z)}{E(Z+1)-E(Z)} \propto \frac{Z-\sigma}{(Z+1-\sigma)^{2}-(Z-\sigma)^{2}} \\
&=\frac{Z-\sigma}{2(Z-\sigma)+1} .
\end{aligned}
$$

For $K$ x-radiations, $\sigma$ is approximately 1 and the last term of (5) reduces to

$$
(Z-1) /(2 Z-1) \text {. }
$$

Over the range of all elements above $Z=10$, this ratio does not vary by more than $2 \frac{1}{2}$ percent.

Figure 1 demonstrates these principles, showing the nominally constant fractional overlap of the calculated half-widths with respect to the $K \alpha$ emission energies and indicating that the counter can resolve the $K$ emission equally well over the range of elements $Z>10$.

It has been shown that the resolution of $x$-rays of adjacent elements is a constant intrinsic property of the proportional counter over all the elements. Beyond that limitation, its use as a spectrometric device for the identification of elements is contingent on the absorption properties of the gas-filling and window and the $x$-ray intensity available from the source.

${ }^{1}$ Curran, Angus, and Cockroft, Phil. Mag. 40, 36 (1949); J. H. Kahn, Oak Ridge National Laboratory Report ORNL-1089, USAEC, Nov. 8, 1951 (unpublished) ; D. West, in Progress in Nuclear Physics (Academic Press, Inc., New York, 1953), Vol. 3.

2 O. R. Frisch, (unpublished).

${ }^{3}$ U. Fano, Phys. Rev. 72, 26 (1947).

4 (To be published).

\section{Molecular Microwave Oscillator and New Hyperfine Structure in the Microwave Spectrum of $\mathrm{NH}_{3} \dagger$}

J. P. Gordon, H. J. Zeiger, ${ }^{*}$ And C. H. Townes Department of Physics, Columbia University, New York, New York (Received May 5, 1954)

A $\mathrm{N}$ experimental device, which can be used as a A very high resolution microwave spectrometer, a microwave amplifier, or a very stable oscillator, has been built and operated. The device, as used on the ammonia inversion spectrum, depends on the emission of energy inside a high- $Q$ cavity by a beam of ammonia molecules. Lines whose total width at half-maximum is six to eight kilocycles have been observed with the device operated as a spectrometer. As an oscillator, the apparatus promises to be a rather simple source of a very stable frequency.

A block diagram of the apparatus is shown in Fig. 1. A beam of ammonia molecules emerges from the source and enters a system of focusing electrodes. These electrodes establish a quadrupolar cylindrical electrostatic 


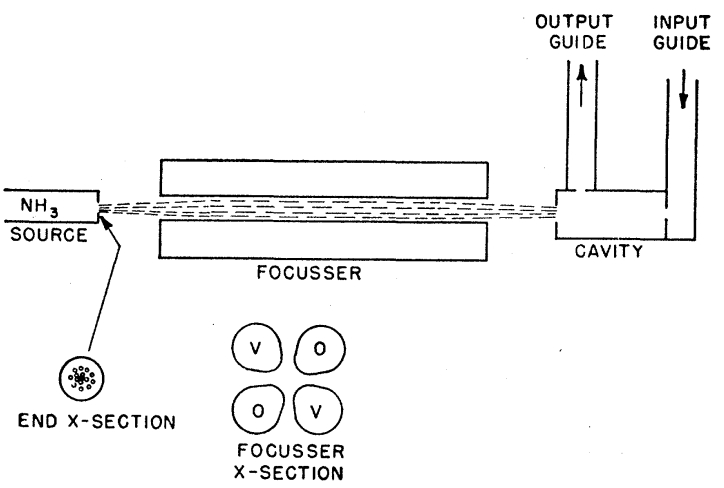

FIG. 1. Block diagram of the molecular beam spectrometer and oscillator.

field whose axis is in the direction of the beam. Of the inversion levels, the upper states experience a radial inward (focusing) force, while the lower states see a radial outward force. The molecules arriving at the cavity are then virtually all in the upper states. Transitions are induced in the cavity, resulting in a change in the cavity power level when the beam of molecules is present. Power of varying frequency is transmitted through the cavity, and an emission line is seen when the klystron frequency goes through the molecular transition frequency.

If the power emitted from the beam is enough to maintain the field strength in the cavity at a sufficiently high level to induce transitions in the following beam, then self-sustained oscillations will result. Such oscillations have been produced. Although the power level has not yet been directly measured, it is estimated at about $10^{-8}$ watt. The frequency stability of the oscillation promises to compare favorably with that of other possible varieties of "atomic clocks."

Under conditions such that oscillations are not maintained, the device acts like an amplifier of microwave power near a molecular resonance. Such an amplifier may have a noise figure very near unity.

High resolution is obtained with the apparatus by utilizing the directivity of the molecules in the beam. A cylindrical copper cavity was used, operating in the TE011 mode. The molecules, which travel parallel to the axis of the cylinder, then see a field which varies in amplitude as $\sin (\pi x / L)$, where $x$ varies from 0 to $L$. In particular, a molecule traveling with a velocity $v$ sees a field varying with time as $\sin (\pi v t / L) \sin (\Omega t)$, where $\Omega$ is the frequency of the rf field in the cavity. A Fourier analysis of this field, which the molecule sees from $t=0$ to $t=L / v$, gives a frequency distribution whose amplitude drops to 0.707 of its maximum at points separated by a $\Delta \nu$ of $1.2 v / L$. The cavity used was twelve centimeters long, and the most probable velocity of ammonia molecules in a beam at room temperature is $4 \times 10^{4} \mathrm{~cm} / \mathrm{sec}$. Since the transition probability is proportional to the square of the field ampli-

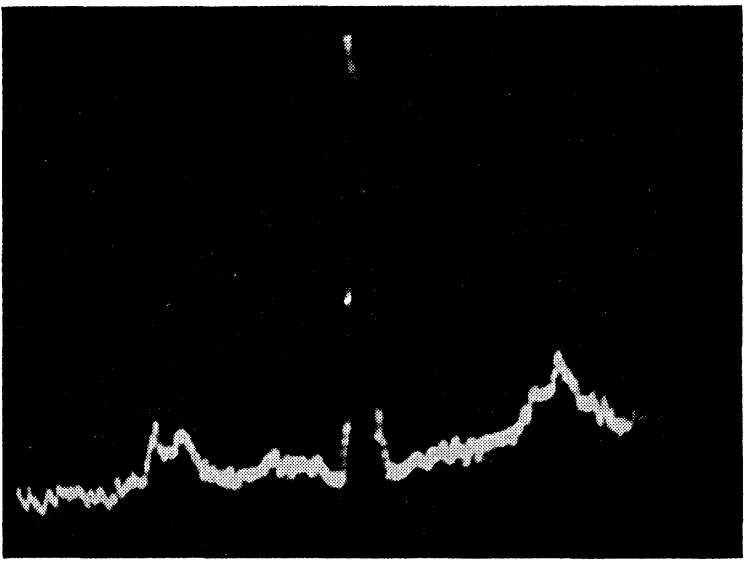

FIG. 2. A typical oscilloscope photograph of the $\mathrm{NH}_{3}, J=K=3$ inversion line at $23870 \mathrm{Mc} / \mathrm{sec}$, showing the resolved magnetic satellites. Frequency increases to the left.

tude, the resulting line should have a total width at half-maximum given by the above expression, which in the present case is $4 \mathrm{kc} / \mathrm{sec}$. The observed line width of $6-8 \mathrm{kc} / \mathrm{sec}$ is close to this value.

The hyperfine structure of the ammonia inversion transitions for $J=K=2$ and $J=K=3$ has been examined, and previously unresolved structure due to the reorientation of the hydrogen spins has been observed. Figure 2 is a typical scope photograph of these new magnetic satellites on the 3,3 line. The observed spectra for the 3,3 line is shown in Fig. 3, which contains all the observed hyperfine structure components, including the quadrupole reorientation transitions of the nitrogen nucleus, which have been previously observed as single lines.

Within the resolution of the apparatus, the hyperfine structures of the upper and lower inversion levels are

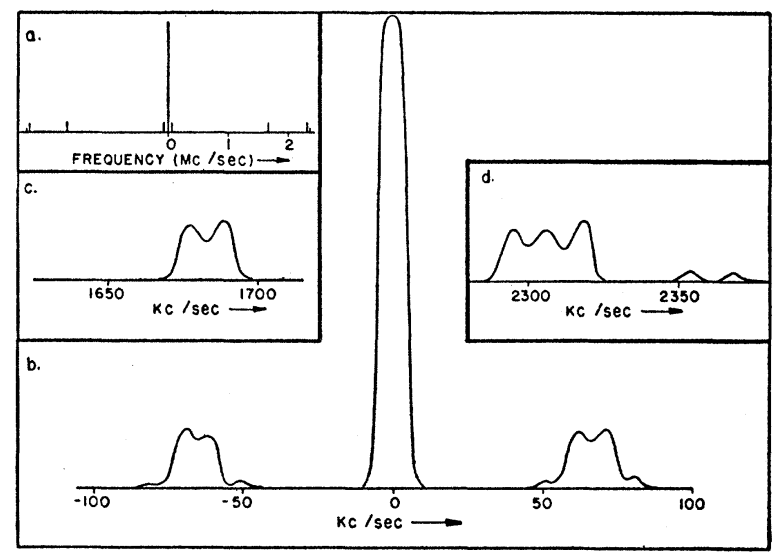

Fig. 3. The observed hyperfine spectrum of the 3,3 inversion line. (a) Complete spectrum, showing the spacings of the quadrupole satellites. (b) Main line with magnetic satellites. (c) Structure of the inner quadrupole satellites. (d) Structure of the outer quadrupole satellites. The quadrupole satellites on the low-frequency side of the main line are the mirror images of those shown, which are the ones on the high-frequency side. 
identical, as evidenced by the fact that the main line is not split. Symmetry considerations require that the hydrogen spins be in a symmetric state under 120degree rotations about the molecular axis. Thus for the 3,3 state, $I_{H}=3 / 2$, and one expects each of the quadrupole levels to be further split into four components by the interaction of the hydrogen magnetic moments with the various magnetic fields of the molecule. At the present writing, the finer details of the expected magnetic splittings have not been worked out.

This type of apparatus has considerable potentialities as a more general spectrometer. Since the effective dipole moments of molecules depend on their rotational state, some selection of rotational states could be effected by such a focuser. Similarly, a focuser using magnetic fields would allow spectroscopy of atoms. Sizable dipole moments are required for a strong focusing action, but within this limitation, the device may prove to have a fairly general applicability for the detection of transitions in the microwave region.

The authors would like to acknowledge the expert help of Mr. T. C. Wang during the latter stages of this experiment.

$\dagger$ Work supported jointly by the Signal Corps, the U. S. Office of Naval Research, and the Air Force.

* Carbide and Carbon post-doctoral Fellow in Physics, now at Project Lincoln, Massachusetts Institute of Technology, Cambridge, Massachusetts.

\section{Oxygen and the Surface Energy-Level Structure on Germanium}

\author{
Edward N. ClaRke \\ Physics Laboratories, Sylvania Electric Products, \\ Bayside, New York \\ (Received April 28, 1954)
}

SURFACE energy levels different from the bulk $S$ levels have been postulated to exist on the germanium surface. ${ }^{1}$ It has also been suggested that both donor- and acceptor-type surface levels exist on germanium. ${ }^{2}$ It is important to know which of these arise from chemical impurities, lattice defects, or the lattice discontinuity at the surface. (The latter are usually referred to as Tamm levels.) In earlier work, we have shown that mechanically disturbing the germanium as by sandblasting produces surface acceptor levels, ${ }^{3}$ and that oxygen may produce surface acceptor levels. ${ }^{4}$ The work to be described provides additional evidence for the role of oxygen and also provides evidence for the presence of a surface donor impurity.

A very thin $\left(10^{-2} \mathrm{~cm}\right)$, low-conductivity $n$ type, etched single crystal of germanium is heated in a vacuum of $10^{-7} \mathrm{~mm} \mathrm{Hg}$. Heating is achieved by passing a large ac current through the crystal. The crystal is then quenched rapidly by shutting off the current, and the conductivity measured. In Fig. 1 the solid lines repre-

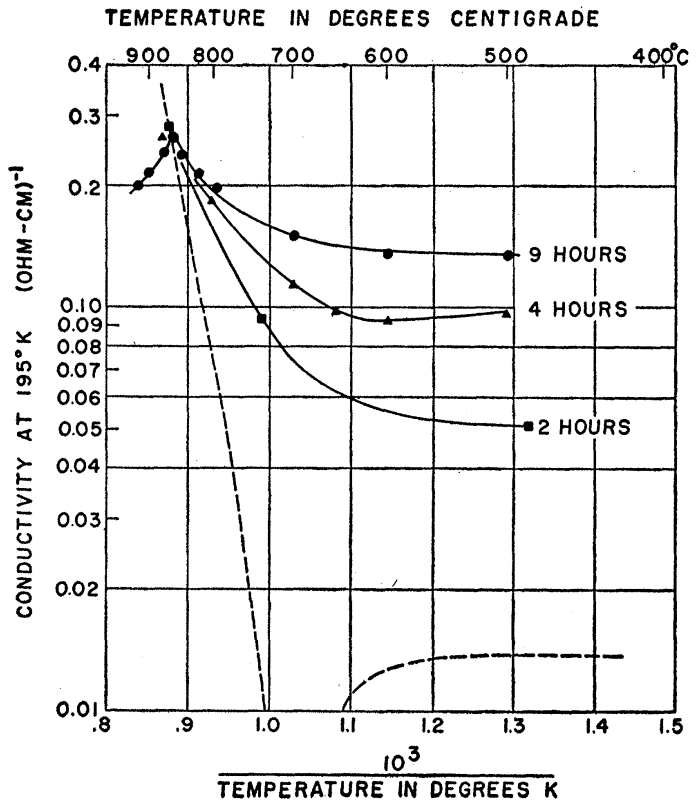

FIG. 1. Conductivity of a thin single crystal of germanium after heating and quenching from various high temperatures in vacuum (vacuum interrupted on several occasions during the experiment to produce the results in Fig. 2). Conductivity of starting material is $0.0137(\mathrm{ohm}-\mathrm{cm})^{-1}$ at $195^{\circ} \mathrm{K}$. Dashed curve represents the effect of Frenkel defects quenched in at the various temperatures.

sent the resulting conductivity, measured at $195^{\circ} \mathrm{K}$, as a function of heat treatment temperature. Similar curves are obtained for conductivity at room temperature. The times 2, 4, and 9 hours refer to the total heating time at temperatures higher than $700^{\circ} \mathrm{C}$ before reaching the corresponding level of conductivity. It is expected that the heat treatment will change the conductivity for two reasons: (1) introduction of Frenkel defects ${ }^{5}$ as shown by the dashed line in Fig. 1, and (2) changes in impurities on the surface. The large excess conductivity actually observed is ascribed to changes in impurities on the surface. Further investigations to be described later show that the excess conductivity, i.e., all of that above the dashed curve, is $n$-type conductivity. The conductivity data thus show that we have either introduced donors or removed acceptors, or both.

That we have removed acceptor levels by heating in vacuum, and that these acceptors result from the presence of oxygen is shown by having the vacuum heated germanium react with oxygen. Figure 2(a) shows conductivity measured at $195^{\circ} \mathrm{K}$ as a function of heating time in oxygen. The oxygen is present at a pressure of about $0.5 \mathrm{~mm} \mathrm{Hg}$. The conductivity decreases markedly with heating in oxygen. We have been able to produce surfaces that react rapidly with oxygen even at room temperature and below. If the germanium is heated once again in vacuum, the conductivity is increased as shown in Fig. 2(b) and can be returned to its initial value by heating at a higher tem- 


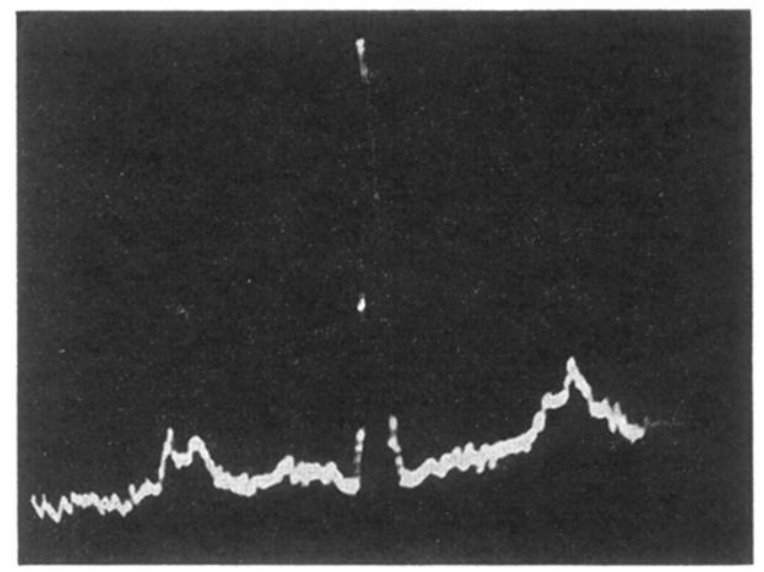

Fig. 2. A typical oscilloscope photograph of the $\mathrm{NH}_{3}, J=K=3$ inversion line at $23870 \mathrm{Mc} / \mathrm{sec}$, showing the resolved magnetic satellites. Frequency increases to the left. 